Received: 24 January 2019

Accepted: 8 April 2019

Online: 10 April 2019

Authors:

S. Patra, P. Mohanty, S. K. Tripathy $₫$ Department of Agricultural Biotechnology, College of Agriculture, OUAT, Bhubaneswar-3, India

$\bigotimes$ swapankumartripathy@gmail.com

Emer Life Sci Res (2019) 5(1): 23-28

E-ISSN: 2395-6658

P-ISSN: 2395-664X

DOI: https://doi.org/10.31783/elsr.2019.512328

\section{Review Article \\ Scope of androgenesis for genetic improvement in rice}

\author{
Sambit Patra, Priyadarshini Mohanty, Swapan K. Tripathy
}

\begin{abstract}
Rice is an autogamous crop. A number of varieties have been bred in this crop using conventional breeding. However, further genetic improvement for seed yield is rarely being achieved as the yield potential has already reached plateau. Recently, frequent disease, pests and insect out-break particularly in areas of intensive cultivation warrants immediate attention. Besides, there is a continuous shift in consumer's preference with the change in the life style. To keep pace with these problems, there is a need to reorient the rice breeding programs in order to short-cut the breeding cycle. Production of double haploids of elite crosses following androgenesis can be a way forward to fine tune the conventional breeding. In this review, the authors stressed upon the use of androgenesis in rice for genetic improvement of tolerance to biotic and abiotic stresses, quality traits and yield per se.
\end{abstract}

Keywords androgenesis, anther culture response, double haploids, rice

\section{Introduction}

Rice (Oryza sativa L.) is the most consumed staple food as a cereal, mostly in Asia. It is also the third-highest produced agricultural commodity [1]. Asian countries have one of the highest population growths which demand increasing production of rice that should be sufficient for maintaining food security. A novel and very efficient method of attaining increased production is the double haploid breeding compared to the time-consuming conventional breeding methods. However, this is still not fully investigated and utilized to augment rice production. Androgenic callus induction is mostly controlled by gametic additive effects than by maternal effects while the reverse is true for green plant regeneration [2]. Harland was first to produce haploid Sea Island cotton, but unfortunately it was not recognized [3]. Blakeslee and co-workers first described the haploid plants - Jimson weed, Datura stramonium [4]. The first in-vitro haploid embryo was developed from excised Datura anther [5-6] and also using rice [7], tobacco [8] and wheat [9]. Further, it was possible to produce completely homozygous DH plants within 8 - 9 months [10] which bypassed the inbreeding process [11] through $\mathrm{F}_{2}$ to $\mathrm{F}_{8}$. Maluszynski reviewed various similar protocols for different plant species [12].

\section{Anther culture techniques}

Double haploids are the result of chromosome doubling of anther culture derived calli by inhibiting the anaphase movement of chromosomes in crop plants [13]. Spontaneous doubling (without colchicines treatment) has been also reported in rice although it was in low frequency [14]. Supplementation of $0.2-0.5 \mathrm{~g} / \mathrm{l}$ colchicines in the media results the development of viable double haploid embryo-like structures (after 24-48h incubation of calli) which on 
transfer to colchicine-free regeneration medium induced as high as $65-70 \%$ viable DH plantlets and it was twice compared to spontaneous doubling. Thus, induced haploidy is proved to be more useful for experimental and commercial uses. Magoon and Khanna [15] listed various methods of inducing haploidy e.g., delayed pollination, use of abortive pollen, temperature extremes, distant hybridization, pollination of $\mathrm{X}$-irradiated of pollen grains and chemical induction. Nakamura obtained double haploid in rice by using abortive pollens [16]. There are various chromosome-doubling chemicals like colchicine, acenapthene vapors, herbicide trifluralin, pronamid, APM and oryzalin. The most commonly used is colchicines as the use of the other chemicals have not been standardized. However, these chemicals are usually not required unless for increasing the double haploid production as the rice haploids usually undergo spontaneous doubling in culture [17]. Moreover, colchicine leads to low seed setting, mixoploids and polyploids development, although these problems could be remedied by application during early androgenic stages [11, 18-19].

Double haploid, also known as double monoploid, are produced from diploids unlike dihaploid which are haploids obtained from tetraploids. Doubled haploids can be used to produce the homozygous lines faster than the conventional inbreeding, usually in one generation [20]. Besides, inbreeds can be developed in just one generation from cross-pollinated crops and self-incompatible crops, which other-wise would require several generations of inbreeding (selfing) to fix homozygosity. Adding different characters to a genotype will take much longer time with conventional approaches and may also be not too successful. However, doubled haploid generation combined with Marker Assisted Selection (MAS) has shown to enhance the efficiency of selection. Additionally, double haploid plants so produced, express dominant and even recessive genes and minor effect genes (QTLs) [21] that improve selection efficiency at early breeding cycle. Besides, a single parent can be loaded with various desirable characters within few years, thus reducing the time consuming steps of selection in recombination breeding.

\section{Factors determining anther culture response}

Haploid induction and further embryo regeneration are determined by several factors [22-23] as follows.

\section{(a) Genotype}

The main constraint in double haploid production through anther culture is the genotype. The indica rice varieties are non-sticky, long-grained and adapted to tropics while the japonica varieties are in vogue sticky, short and bold-grained and are grown in temperate and tropical uplands. Japonica group inherently possesses better response to callus induction and plantlet regeneration from anthers than indica types [24]. But, this becomes a major hindrance in genetic improvement of indica rice [25], although a number of japonica varieties have been developed in japonica types [26] through anther culture. Besides, lower frequencies, necrosis of anther [27] and the formation of higher frequency of albino plants [27-28] further restrict the use of such novel technique in indica rice. It is worth to note that only one out of 35 indica varieties showed pollen callusing on N6 medium [29].

\section{(b) Physiological status of donor plants}

The physiology of the donor plant has been found to play an important role in determining the future development of the double haploid plants. The anthers collected from plants grown under moderate moisture stress with low nitrogen fertilizer application promote anther culture response. Temperature during the booting stage also influences the quality of anther. Moreover, 12 hour photoperiod and $34{ }^{\circ} \mathrm{C} / 25^{\circ} \mathrm{C}$ day/night temperature at booting stage are favorable for better anther response for callus induction and plant regeneration. Anthers selected from donors grown in open fields are reported to offer better response to in vitro culture than those grown indoors [30]. Additionally, anthers from the primary tillers produced more callusing response than those from the side tillers in rice.

\section{(c) Stage of anther development}

The pollens at early to mid-uninucleate stage and definitely not at tetrad stage are most preferred for androgenesis. Bisnoi and co-workers have suggested using the distance between the flag leaf to leaf 
auricle to measure the quality of pollen for culturability in rice and it correspond to the $3-4 \mathrm{~cm}$ distance between the collar of the flag leaf and ligule of the penultimate leaf [31].

\section{(d) Stress pre-treatment of anthers}

Either osmotic stress (mannitol), mild heat $\left(30-40{ }^{\circ} \mathrm{C}\right)$ or chemical or more specifically, the cold pretreatment of anthers is needed to initiate sporophytic growth [28, 32-33]. Mishra and Rao have listed various studies on stress pre-treatment of anthers to induce callusing response [23]. Cold treatment stops gamete formation and this in turn has a positive effect on callus formation. However, as a result, the chlorophyll being degraded leads to formation of albino plants. Cold treatment led to a delayed effect on anther wall senescence, along with the increased symmetric division of pollen grains. Amino acids and shock-thermic proteins were also released in addition to other compounds that help in androgenesis [34]. Cold pre-treatment for 14 days at $8{ }^{\circ} \mathrm{C}$ is shown to be the most effective for anther culture in selected varieties of indica, japonica rice and some inter sub-specific hybrids [35]. Mishra et al., [17] reported a pretreatment at $10^{\circ} \mathrm{C}$ for 7-9 $\mathrm{d}$ having a positive effect on indica rice hybrids. Treatments of this type further corroborated the positive results of treatment of callus in 13 indica rice genotypes at $12^{\circ} \mathrm{C}$ for 5 days [21]. Similarly, heat pre-treatment showed a positive effect by disrupting the development of anther somatic tissue (tapetum and anther wall) and encouraging the callus induction from anthers [36-37]. Interestingly, rice anthers exposed to high temperature during meiosis led to albinism. However, a specific optimal temperature pre-treatment of anthers is necessary for follow-up callus development and plantlet regeneration that is reported to be genotype dependent [38].

\section{(e) In vitro stress treatment during culture process}

In vogue, the regeneration frequency decreases in presence of $\mathrm{NaCl}$, but an opposite effect may be observed on regenerated plantlets. Tryptophan exerts a positive effect on regeneration and on the survival rates of cultivars under $\mathrm{NaCl}$ stress. In general, both abscisic acid (ABA) and polyethylene glycol (PEG) delay the regeneration process and reduce the mean number of plantlets obtained per regenerating callus. ABA and PEG both have negative effects on rooting ability and survival of the regenerated plantlets in indica genotype, but have not been found to affect the regeneration percentages. Besides, there seems to be no effect of proline application regardless of $\mathrm{NaCl}$ dosage or cultivar.

\section{(f) Media and phytohormones}

Nitrogen promotes development of superior microspores with high embryogenic activity [39]. The most common medium for development of androgenic callus is N6 and MS- medium + 2,4-D(2-3mg/l) with some variations (higher nitrogen and iron concentration) as needed [40], while MS medium, supplemented with BAP/Kinetin with lower concentration of NAA, is generally used for plant regeneration. Various experiments conducted by different researchers have also shown that application of polyamines promotes the plantlet regeneration [33]. It was observed that IAA reduced the shoot formation, but increased the root development [41]. Maltose has been reported to be more suitable as a media for androgenesis in comparison to sucrose. It also minimizes the chances of albino production in various cereal species [42-43].

\section{Double haploid breeding in rice}

$\mathrm{Ba}$ Bong and Swaminamthan [44] conducted a breeding program for testing the hybrid vigor using the double haploid rice. A few DH lines derived from anther culture of hybrids of genetically diverse parents could reach the heterotic level of the hybrid [44] which further pinpoints the prospect of anther culture technique in rice. DH technology has been used for the production of more than 100 rice varieties [45-46] with characteristics like superior grain quality; biotic-resistance (blast, bacterial blight, and brown planthopper) and various abiotic stress tolerances. DH lines from cv. Calrose 76 showed variation with improved agronomic characters e.g., seed number and size, panicle size, plant height and tiller number [47] and improved protein content and higher yields [48]. Xa and Lang [49] recovered 133 DH lines out of 
which 22 outstanding DH lines were selected for yield and grain quality. Rasoazanakolona [50] reported nutritional benefits e.g., higher starch and ash content, higher quantity of desired minerals, but lower lipid quantity along with lower sugar and protein content in DH lines of rice. This seems to be a way forward towards biofortification of nutrients, while the decrease in lipid quantity in rice grains would help for longer storage.

\section{Conclusion and future prospects}

Double haploidy is an innovative method to obtain plants that are agronomically superior to the parental type. Though this technology is promising, various technological advances need to be refined for its commercialization. Common problems of anther culture technology include albinism and failure during the various developmental stages exclusively for fertile DH production. Further experiments and research are required for overcoming these obstacles, which would benefit rapid genetic improvement in rice to keep the pace with diseases and insects/pests out break and changing the consumer preference.

\section{Acknowledgements}

The authors highly acknowledge the contributions of different researchers included in the text as references.

\section{References}

[1] FAOSTAT (2017). UN Food and Agriculture Organization, Corporate Statistical Database. Retrieved November 9, 2018.

[2] J. Yan, Q. Xue and J. Zhu (1996). Genetic studies of anther culture ability in rice (Oryza sativa). Plant Cell Tissue Organ Cult., 45: 253-258.

[3] S. C. Harland (1920). A note on a peculiar tribe of rogue in Sea Island cotton. Agr. News, Barbados, 19, 29.

[4] A. F. Blakeslee, J. Belling, M. E. Farnham and A. D. Bergner (1922). A haploid mutant in the Jimson weed, Datum stramonium. Science, 55: 646-647.

[5] S. Guha and S. C. Maheshwari (1964). In vitro production of embryos from anthers of Datura. Nature, 204: 497.

[6] S. Guha and S.C. Maheshwari (1966). Cell division and differentiation of embryos in the pollen grains of Datura in vitro. Nature, 212: 97-98.

[7] H. Niizeki and K. Oono (1968). Induction of haploid rice plant from anther culture Proc Japan Acad., 44: 554-557.

[8] J. P. Nitsch and C. Nitsch (1969). Haploid plants from pollen grains. Science, 163:85-87.

[9] B. P. Forster, E. Heberle-Bors, K. J. Kasha and A. Touraev (2007). The resurgence of haploids in higher plants. Trends Plant Sci, 12: 368-375.

[10] S. Agache, B. Bachelier, J. de Buyser, Y. Henry and J. Snape (1989). Genetic analysis of anther culture response in wheat using aneuploid, chromosome substitution and translocation lines. Theor Appl Genet., 77: 7-11.

[11] M. A. Germana (2011). Anther culture for haploid and doubled haploid production, Plant Cell Tissue Organ Cult., 104: 283-300.

[12] M. Maluszynski; K. J. Kasha and I. Szarejko (2003). Published doubled haploid protocols in plant species. In Doubled haploid production in crop plants. pp. 309-335. Springer, Dordrecht.

[13] H. Hu and J. Z. Zeng (1984). Development of new varieties via anther culture. (No. 86-043649. CIMMYT.).

[14] P. Premvaranon, S. Vearasilp, S. N. Thanapornpoonpong, D. Karladee, S. Gorinstein (2011). In vitro studies to produce double haploid in Indica hybrid rice, Biologia, 66: 1074-1081.

[15] M. L. Magoon and K. R. Khanna (1963). Haploids. Caryologia, 16: 191-235.

[16] S. Nakamura (1933). The haploid plant in rice. Jap. Jour. Gen., 8: 223-227. 
[17] M. Rukmini, G. J. N. Rao and R. N. Rao (2013) Effect of cold pretreatment and phytohormones on anther culture efficiency of two Indica rice (Oryza sativa L.) hybrids: Ajay and Rajalaxmi. Experimental Biol. Agricultural Sci., 1: 69-76.

[18] S. K. Tripathy (2018). Anther culture for double haploid breeding in rice-a way forward, Rice Genomics and Genetics, 9: 1-6.

[19] A. M. Castillo, L. Cistué, M. P. Vallés, and M. Soriano (2009). Chromosome doubling in monocots. In Advances in haploid production in higher plants. pp329-338. Springer, Dordrecht.

[20] S. M. Jain, S. K. Sopory and R. Veilleux (Eds.). (1996). In Vitro Haploid Production in Higher Plants: Volume 2: Applications, Vol. 24. Springer Science and Business Media.

[21] L. Kaushal, R. Sharma, S. M. Balachandran, K. Ulaganathan and V. Shenoy (2014). Effect of cold pretreatment on improving anther culture response of rice (Oryza sativa L.). J. Exp. Biol. Agric. Sci., 2: 233-242.

[22] J. Murovec and B. Bohanec (2012). Haploids and doubled haploids in plant breeding. In Plant breeding. IntechOpen. doi: $10.5772 / 29982$.

[23] R. Mishra, G. J. N. Rao (2016). In-vitro Androgenesis in Rice: Advantages, Constraints and Future Prospects. Rice Science, 23: 57-68.

[24] W. T. B. Thomas, B. Gertson and B. P. Forster (2003). Doubled haploids in breeding. In Doubled Haploid Production in Crop Plants (pp. 337-349). Springer, Dordrecht.

[25] I. S. Dewi, B. S. Purwoko, H. Aswidinnoor, I. H. Somantri and M. A. Chozin (2009). Plant regeneration from anther cultures of several genotypes of Indica rice tolerant to aluminum toxicity. Indonesian $\mathbf{J}$ Agric., 2: 1-5.

[26] D. Grewal, C. Manito and V. Bartolome (2011). Doubled Haploids Generated through Anther Culture from Crosses of Elite Indica and Japonica Cultivars and/or Lines of Rice: Large-Scale Production, Agronomic Performance, and Molecular Characterization. Crop Sci., 51: 2544-2553.

[27] S. S. Gosal, A. S. Sindhu, J. S. Sandhu, R. Sandhu-Gill, B. Singh, G. S. Khera and G. S. Sidhu et al., (1997). Haploidy in rice. In: S. M. Jain, S. K. Sopory and R. E. Veilleux (eds) Cereals, In Vitro Haploid Production in Higher Plants, 1-35. Kluwer Academic Publishers, Dordrecht

[28] T. D. Silva (2010). Indica rice anther culture: Can the impasse be surpassed? Plant Cell Tissue Organ Cult., 100: 1-11.

[29] Z. Lentini, P. Reyes, C. P. Martinez, W. M. Roca (1995). Androgenesis of highly recalcitrant rice genotypes with maltose and silver nitrate Plant Sci., 110:127-138.

[30] R. Veeraraghavan (2007). A study on the comparison of anther culture response in different varieties of rice Oryza sativa L. subspecies Indica. University of Colombo, Colombo, Sri Lanka.

[31] U. Bishnoi, R. K. Jain, J. S. Rohilla, V. K. Chowdhury, K. R. Gupta and J. B. Chowdhury (2000). Anther culture of recalcitrant indica $\times$ Basmati rice hybrids. Euphytica, 114: 93-101.

[32] S. K. Datta and G. Wenzel (1988). Single microspore derived embryogenesis and plant formation in barley (Hordeum vulgare L.). Archiv fuer. Zuechtungsforschung (German DR).

[33] S. Cha-Um and B. Srianan, A. Pichakum and C. Kirdmanee (2009). An efficient procedure for embryogenic callus induction and double haploid plant regeneration through anther culture of Thai aromatic rice (Oryza sativa L. subsp. indica). In Vitro Cell. Dev. Biol.- Plant, 45:171-179.

[34] E. Kiviharju and E. Pehu (1998). The effect cold and heat pretreatments on anther culture response of Avena sativa and A. sterilis. Plant Cell Tiss Organ Cult., 54: 97-104.

[35] H. M. I. Herath, D. C. Bandara, P. K. Samarajeewa and D. S. A. Wijesundara (2009). Effect of low temperature pre-treatment on anther culture in selected indica, japonica rice varieties and their inter subspecific hybrids. Cey. J. Sci. (Bio. Sci.), 38: 11-16.

[36] J. M. Dunwell, M. Cornish, A. G. L. de Courcel and J. E. Middlefelll-Williams (1983). Induction and growth of 'microspore-derived' embryo of Brassica napus ssp. oleifera. J. Exp. Bot. 34: 1768-1778.

[37] A. M. R. Ferrie and W. A. Keller (1995). Development of methodology and applications of doubled haploids in Brassica rapa. In: Proceedings of the 9th International Rapeseed Congress. Pp 807-809. GCIRC, Cambridge. 
[38] J. W. Ouyang, S. M. Zhou, S. E. Jia (1983). The response of anther culture to culture temperature in Triticum aestivum. Theor Appl Genet., 66: 101-109.

[39] P.C. Lapitan, V. N. Villegas (1999). Anther culture response of some genotypes with varying amylase content to nitrogen fertilization. Philippine Journal of Crop Sci., 24: 79-84.

[40] C. Chih-Ching, W. Ching-Chu, S. Ching-San, H. Chen, Y. Kwang-Chu,C. Chih-Yin and B. Feng-Yun (1975). Establishment of an efficient medium for anther culture of rice through comparative experiments on the nitrogen sources. Scientia Sinica. 18: 659-668.

[41] S. Lutts, J. M. Kinet and J. Bouharmont (1999). Improvement of rice callus regeneration in the presence of NaCl. Plant Cell Tissue Organ Cult., 57: 3-11.

[42] C. Sen, R. P.Singh, M. K. Singh and H. B. Singh (2011). Effect of cold pretreatment on anther culture of boro rice hybrids. Int. J. Plant Rep. Biol., 3: 69-73.

[43] S. G. Park, M. Ubaidillah and K.-M. Kim (2013). Effect of maltose concentration on plant regeneration of anther culture with different genotypes in rice (Oryza sativa L.). American Journal of Plant Sci., 4(11): 2265-2270.

[44] B. Ba Bong and M. S. Swaminathan (1995). Magnitude of hybrid vigor retained in double haploid lines of some heterotic rice hybrids. Theor. Appl. Genet., 90: 253-257.

[45] F. J. Zapata-Arias (2003). Laboratory protocol for anther culture technique in rice. In Doubled Haploid Production in Crop Plants. pp109-116. Springer, Dordrecht.

[46] R. Siddique (2015). Impact of different media and genotypes in improving anther culture response in rice (Oryza sativa) in Bangladesh. European Scientific J., 11: 164-169.

[47] G. W. Schaeffer (1982). Recovery of heritable variability in anther derived double haploid rice. Crop Sci., 22: 1160-1164.

[48] G. W. Schaeffer, F. T. Jr Sharpe and P. B. Creagan (1984). Variation for improved protein and yield from rice anther culture. Theor Appl Genet., 67: 383-389.

[49] T. T. Xa and N. T. Lang (2011). Rice breeding for high grain quality through anther culture. Omonrice, 18: $68-72$.

[50] V. Rasoazanakolona, B. B. Rabealaina, A. Andrianjaka, X. Rakotonjanahary, R. S. Sangwan, and N. V. Rakotoarisoa (2016). Evaluation of Grain Quality and Nutritional Quality of Double Haploid DHP6, An Elite Rice Line in Madagascar, Proc. Latvian Acad. Sci. Section B, 70: 378-383. 